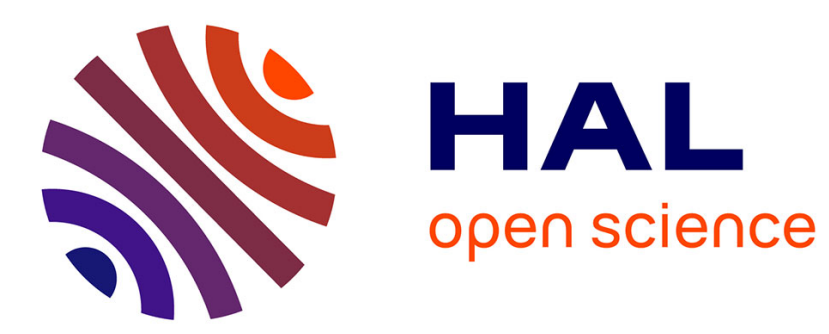

\title{
A Theoretical Study of the Biselective Double Pulsed Field Gradient Spin-Echo NMR Experiment
}

Jean-Marc Nuzillard

\section{To cite this version:}

Jean-Marc Nuzillard. A Theoretical Study of the Biselective Double Pulsed Field Gradient Spin-Echo NMR Experiment. Molecular Physics, 2008, 105 (17-18), pp.2243-2250. 10.1080/00268970701530975 . hal-00513125

\section{HAL Id: hal-00513125 \\ https://hal.science/hal-00513125}

Submitted on 1 Sep 2010

HAL is a multi-disciplinary open access archive for the deposit and dissemination of scientific research documents, whether they are published or not. The documents may come from teaching and research institutions in France or abroad, or from public or private research centers.
L'archive ouverte pluridisciplinaire HAL, est destinée au dépôt et à la diffusion de documents scientifiques de niveau recherche, publiés ou non, émanant des établissements d'enseignement et de recherche français ou étrangers, des laboratoires publics ou privés. 


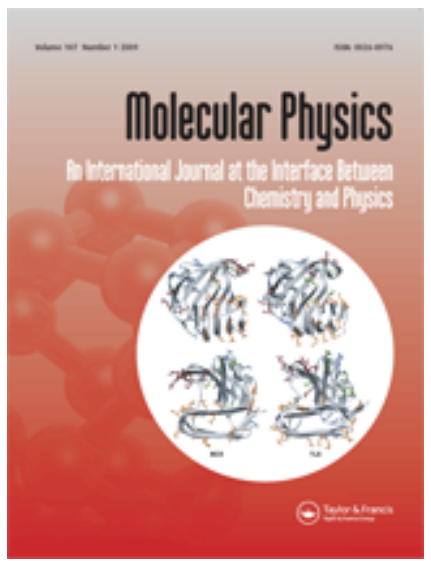

A Theoretical Study of the Biselective Double Pulsed Field Gradient Spin-Echo NMR Experiment

\begin{tabular}{|c|c|}
\hline Journal: & Molecular Physics \\
\hline Manuscript ID: & TMPH-2007-0120 \\
\hline Manuscript Type: & Full Paper \\
\hline $\begin{array}{r}\text { Date Submitted by the } \\
\text { Author: }\end{array}$ & 11-May-2007 \\
\hline Complete List of Authors: & $\begin{array}{l}\text { Nuzillard, Jean-Marc; University of Reims, Institut de Chimie } \\
\text { Moléculaire }\end{array}$ \\
\hline Keywords: & Liquid state NMR, selective pulses, static field gradients, spin echo \\
\hline \multicolumn{2}{|c|}{$\begin{array}{l}\text { Note: The following files were submitted by the author for peer review, but cannot be converted } \\
\text { to PDF. You must view these files (e.g. movies) online. }\end{array}$} \\
\hline mph1.tex & \\
\hline
\end{tabular}

\section{scholarONE" \\ Manuscript Central}




\title{
A Theoretical Study of the Biselective Double Pulsed Field Gradient Spin-Echo NMR Experiment
}

\author{
Jean-Marc Nuzillard* \\ Institut de Chimie Molculaire de Reims, BP 1039, 51687 REIMS Cedex 2, France.
}

(xx May 2007)

\begin{abstract}
The application of a biselective radiofrequency pulse to a coupled homonuclear spin system produces effets that are exploited in liquid-state NMR experiments. The insertion of such an RF pulse in a double pulsed field gradient spin echo sequence results in a module for the biselective production of antiphase magnetization states. Biselectivity is obtained through cosine modulation of a singly selective pulse profile. A full description of the module behavior is possible by means of four parameters, among which two are related to a property that was named "pulse ellipticity". The theory is in good agreement with the experimental results and numerical simulations. It is thus possible to predict how a maximum of antiphase magnetization can be produced.
\end{abstract}

Keywords: Liquid state NMR; selective pulses; static field gradients; spin echo

\section{Introduction}

The simultaneous application of multiplet selective pulses to two mutually coupled spins is known to produce effects that are different from those observed with non coupled spins. These effects have been studied in double inversion and double excitation experiments [1-3]. The underlying theory was first presented by Emsley et al [1], and then by Blechta et al [3] and Zhou et al in a more general context [4]. Later, the inclusion of simultaneous selective pulses in pulsed field gradient spin echo (DPFGSE) experiments [5] led to doubly selective experiments [6]. They allowed the extraction of two signals from a crowded spectrum if, and only if, they originate from two scalarly coupled spins. Further spin manipulation led to doubly selective TOCSY and TOCSY-COSY experiments [6].

In the basic pulse sequence (see Fig. 1), the $\delta$ delays are adjusted so that a maximum of antiphase magnetization is produced just before the last two hard pulses that act as a double quantum filter. The only detected signals are those that arise from nuclei pairs whose transverse magnetization is subjected to simultaneous selective refocusing.

The main goal of this article is to theoretically establish the relationship between the duration of the $\delta$ delays and $f(\delta)$, the intensity of the recorded signal of an IS spin system. The resonance frequency difference of I and S, the value $J$ of their scalar coupling and the shape of the soft pulses are the parameters of the problem. The result is validated by the experiment in Fig. 1, carried out on the AX system of ethylenic protons in cinnamic acid.

*Corresponding author. Email: jm.nuzillard@univ-reims.fr

Molecular Physics

ISSN 00268976 print/ISSN 13623028 online @ 2007 Taylor \& Francis http://www.tandf.co.uk/journals DOI: $10.1080 / 0026897 \mathrm{YYxxxxxxxx}$ 
$\Delta$

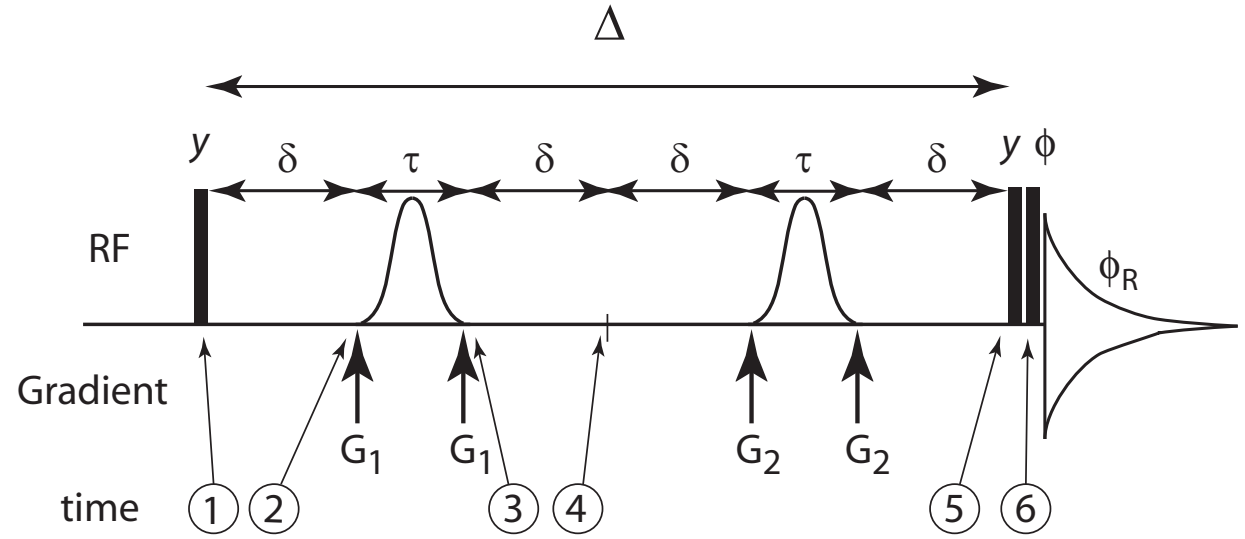

Figure 1. Pulse sequence for the biselective extraction of a coupled spin pair. Thin black bars are all hard $90^{\circ}$ pulse. The phase programme is $\phi=x,-y,-x, y$ and $\phi_{R}=x, y,-x,-y$. The bell shaped curves are soft pulses that simultaneously refocus the transverse magnetization of the spin pair.

\section{Theory}

The theoretical part of this article deals with a weakly coupled homonuclear IS spin system that undergoes the pulse sequence in Fig. 1. The transmitter frequency of the spectrometer is assumed to be set at the mean of the resonance frequencies of nuclei I and S. Their difference $\Delta \nu=\left|\nu_{I}-\nu_{S}\right|$ is noted $2 \nu_{0}$. Therefore, in the rotating frame of reference, the angular frequencies of their magnetization precession $\Omega_{I}$ and $\Omega_{S}$ are related by:

$$
\Omega_{I}=-\Omega_{S}=\omega_{0}=2 \pi \nu_{0}
$$

The density matrix of the spin system at time $t_{1}$ is

$$
\sigma_{1}=I_{x}+S_{x}=\frac{1}{2}\left(I_{-}+S_{-}\right)+\frac{1}{2}\left(I_{+}+S_{+}\right)
$$

The evolution of -1 and +1 quantum states will be simultaneously considered because they are very closely related. During the $\delta$ delays, $I_{-}+S_{-}$evolves under the action of the Hamiltonian operator

$$
\mathcal{H}_{0}=\pi J 2 I_{z} S_{z}+\omega_{0}\left(I_{z}-S_{z}\right)
$$

in the -1 quantum space that is spanned by elements in the $\mathcal{B}^{-}$base:

$$
\mathcal{B}^{(-)}=\left\{I_{-}+S_{-}, 2 I_{-} S_{z}+2 I_{z} S_{-}, I_{-}-S_{-}, 2 I_{-} S_{z}-2 I_{z} S_{-}\right\}
$$

At time $t_{2}$, the field gradient pulse spatially labels the sample magnetization so that only +1 quantum states are recovered after the second gradient pulse. The spin state $\sigma_{3}$ at $t_{3}$ (at least for the components that finally produce detectable magnetization) is therefore a combination of elements in the $\mathcal{B}^{(+)}$base:

$$
\mathcal{B}^{(+)}=\left\{I_{+}+S_{+}, 2 I_{+} S_{z}+2 I_{z} S_{+}, I_{+}-S_{+}, 2 I_{+} S_{z}-2 I_{z} S_{+}\right\}
$$

During the second $\delta$ delay, under action of $\mathcal{H}_{0}, \sigma_{3}$ evolves in the subspace of the +1 quantum states until $t_{4}$. Between $t_{4}$ and $t_{5}$, the event sequence is identical to the one between $t_{1}$ and $t_{4}$. The +1 quantum states are converted back to -1 quantum states, assuming that the second gradient pair does not bring back magnetization 
that was defocused by the first gradient pair. The following development successively focuses on what happens between $t_{2}$ and $t_{3}, t_{1}$ and $t_{4}, t_{1}$ and $t_{5}$, and between $t_{1}$ and $t_{6}$.

\subsection{Between $t_{2}$ and $t_{3}$}

The Hamiltonian operator that acts between times $t_{2}$ and $t_{3}$ is

$$
\mathcal{H}_{1}=\mathcal{H}_{0}+\mathcal{H}^{\mathrm{rf}}
$$

with

$$
\mathcal{H}^{\mathrm{rf}}=\omega_{1}(t)\left(I_{x}+S_{x}\right)
$$

and

$$
\omega_{1}(t)=2 \omega_{1}^{m}(t) \cos \left(\omega_{0}\left(t-\frac{\tau}{2}\right)\right)=2 \omega_{1}^{m}(t) \cos \left(\omega_{0} t^{\prime}\right)
$$

where $\omega_{1}^{m}(t)$ defines the shape of the single site selective pulse from which the two-site selective pulse is defined. The time variable $t^{\prime}$ is introduced so that $t^{\prime}=$ 0 corresponds to the middle of the soft inversion pulse. The shape of the pulse envelope, $\omega_{1}^{m}(t)$, is assumed to be symmetrical. The $\cos \left(\omega_{0} t^{\prime}\right)$ factor creates the excitation sidebands at offsets $+\omega_{0}$ and $-\omega_{0}$ that simultaneously affect the I and $\mathrm{S}$ nuclei. The expression of $\mathcal{H}^{\mathrm{rf}}$ can be split into two terms:

$$
\begin{aligned}
\mathcal{H}^{\mathrm{rf}} & =\left[\omega_{1}^{m} \cos \left(\omega_{0} t^{\prime}\right)\left(I_{x}+S_{x}\right)+\omega_{1}^{m} \sin \left(\omega_{0} t^{\prime}\right)\left(I_{y}-S_{y}\right)\right] \\
& +\left[\omega_{1}^{m} \cos \left(\omega_{0} t^{\prime}\right)\left(I_{x}+S_{x}\right)-\omega_{1}^{m} \sin \left(\omega_{0} t^{\prime}\right)\left(I_{y}-S_{y}\right)\right]
\end{aligned}
$$

The second bracketed term describes a radiofrequency field that rotates at frequency $-\omega_{0}$ for the I nucleus and at $+\omega_{0}$ for the $\mathrm{S}$ one. The hypothesis

$$
\left|\omega_{1}^{m}\right| \ll 2 \omega_{0}
$$

is made to ensure that the radiofrequency sideband at the frequency of the I resonance does not affect the S nucleus, and vice versa. Consequently, the Hamiltonian operator that drives the evolution of the system between $t_{2}$ and $t_{3}$ can be transformed to $\mathcal{H}_{1}$ :

$$
\begin{aligned}
\mathcal{H}_{1}(t) & =\pi J 2 I_{z} S_{z}+\omega_{0}\left(I_{z}-S_{z}\right) \\
& +\omega_{1}^{m}(t)\left[\left(\cos \left(\omega_{0} t^{\prime}\right) I_{x}+\sin \left(\omega_{0} t^{\prime}\right) I_{y}\right)\right] \\
& +\omega_{1}^{m}(t)\left[\left(\cos \left(\omega_{0} t^{\prime}\right) S_{x}-\sin \left(\omega_{0} t^{\prime}\right) S_{y}\right)\right]
\end{aligned}
$$

in which the spin operators of I and S are separated, with the exception of for their shared coupling interaction.

The expression of $\mathcal{H}_{1}(t)$ is made independent of $\omega_{0}$ by means of the similarity transformation $[1,7]$ that is defined by the unitary operator $U(t)$ :

$$
U(t)=e^{i \omega_{0}\left(I_{z}-S_{z}\right) t^{\prime}}
$$


The evolution of any initial state $\sigma(0)$ to the final state $\sigma(\tau)$ by application of $\mathcal{H}_{1}(t)$ is calculated in three steps :

$$
\begin{aligned}
\sigma(0) \longrightarrow & \sigma^{r}(0)=U(0) \sigma(0) U^{-1}(0) \\
\stackrel{\mathcal{H}^{r}}{\longrightarrow} & \sigma^{r}(\tau) \\
& \longrightarrow U^{-1}(\tau) \sigma^{r}(\tau) U(\tau)
\end{aligned}
$$

in which

$$
\mathcal{H}^{r}=U \mathcal{H}_{1} U^{-1}-i U \frac{d U^{-1}}{d t}=\pi J 2 I_{z} S_{z}+\omega_{1}^{m}(t)\left(I_{x}+S_{x}\right)
$$

$\mathcal{H}^{r}$ is still time-dependent if $\omega_{1}^{m}(t)$ is not constant. However, the selective pulse is implemented as a series of $N$ constant field strength subpulses of length $\varepsilon=\tau / N$ during which the spin state evolution can be calculated at times $\tau_{1}, \tau_{2}, \ldots \tau_{N}=\tau$.

$$
\sigma^{r}(\tau)=e^{-i \mathcal{H}^{r}\left(\tau_{N}\right) \varepsilon} \ldots e^{-i \mathcal{H}^{r}\left(\tau_{1}\right) \varepsilon} \sigma^{r}(0) e^{+i \mathcal{H}^{r}\left(\tau_{N}\right) \varepsilon} \ldots e^{+i \mathcal{H}^{r}\left(\tau_{1}\right) \varepsilon}
$$

If $\sigma(0)$ is a -1 quantum state, so is $\sigma^{r}(0)$. The evolution of the state of an IS spin system under the action of the constant Hamiltonian operator

$$
\mathcal{H}_{k}=\pi J 2 I_{z} S_{z}+\omega_{1}^{m}\left(\tau_{k}\right)\left(I_{x}+S_{x}\right)
$$

during a time $\varepsilon$ is fully described in references [3] and [4]. The relevant results are reported hereafter.

The spin states independently evolve in four subspaces that are spanned by four sets of base elements:

$$
\begin{aligned}
\mathcal{B}^{(2)} & =\left\{I_{x}-S_{x}, 2 I_{y} S_{z}-2 I_{z} S_{y}\right\} \\
\mathcal{B}^{(3)} & =\left\{I_{x}+S_{x}, 2 I_{y} S_{z}+2 I_{z} S_{y}, 2 I_{z} S_{z}-2 I_{y} S_{y}\right\} \\
\mathcal{B}^{(4 S)} & =\left\{I_{y}+S_{y}, 2 I_{x} S_{z}+2 I_{z} S_{x}, I_{z}+S_{z}, 2 I_{x} S_{y}+2 I_{y} S_{x}\right\} \\
\mathcal{B}^{(4 A)} & =\left\{I_{y}-S_{y}, 2 I_{x} S_{z}-2 I_{z} S_{x}, I_{z}-S_{z}, 2 I_{x} S_{y}-2 I_{y} S_{x}\right\}
\end{aligned}
$$

In these bases, the matrices of the superoperator $\hat{\mathcal{H}}_{k}$ that is defined by

$$
\sigma\left(\tau_{k}\right)=e^{-i H_{k} \varepsilon} \sigma\left(\tau_{k-1}\right) e^{+i H_{k} \varepsilon}=\hat{\mathcal{H}}_{k}\left(\sigma\left(t_{k-1}\right)\right)
$$

are $R_{k}^{(2)}, R_{k}^{(3)}, R_{k}^{(4 S)}$ and $R_{k}^{(4 A)}$ :

$$
\begin{aligned}
& R_{k}^{(2)}=\left[\begin{array}{cc}
\cos (\pi J \varepsilon) & -\sin (\pi J \varepsilon) \\
\sin (\pi J \varepsilon) & \cos (\pi J \varepsilon)
\end{array}\right] \\
& R_{k}^{(3)}=\left[\begin{array}{ccc}
a^{2}+b^{2}-c^{2} & -2 a c & 2 b c \\
2 a c & a^{2}-b^{2}-c^{2} & -2 a b \\
2 b c & 2 a b & a^{2}-b^{2}+c^{2}
\end{array}\right] \\
& R_{k}^{(4 S)}=R_{k}^{(4 A)}=\left[\begin{array}{cccc}
a d-c e & a e+c d & -b d & b e \\
-a e-c d & a d-c e & b e & b d \\
b d & b e & a d+c e-a e+c d \\
b e & -b d & a e-c d & a d+c e
\end{array}\right]
\end{aligned}
$$


with

$$
\begin{array}{rlrl}
\omega_{\mathrm{eff}}^{2} & =\omega_{1}^{m}\left(\tau_{k}\right)^{2}+\left(\frac{\pi J}{2}\right)^{2} & a & =\cos \left(\omega_{\mathrm{eff}} \varepsilon\right) \\
d & =\cos \frac{\pi J \varepsilon}{2} & b & =\frac{\omega_{1}^{m}\left(\tau_{k}\right)}{\omega_{\mathrm{eff}}} \sin \left(\omega_{\mathrm{eff}} \varepsilon\right) \\
e & =\sin \frac{\pi J \varepsilon}{2} & c & =\frac{\pi J}{2 \omega_{\mathrm{eff}}} \sin \left(\omega_{\mathrm{eff}} \varepsilon\right)
\end{array}
$$

The matrices $R^{(2)}, R^{(3)}$, and $R^{(4)}=R^{(4 S)}=R^{(4 A)}$ of the global superoperator $\hat{\mathcal{H}}^{r}$ that act between $t_{2}$ and $t_{3}$ are the products of the subpulse matrices:

$$
R^{(l)}=R_{N}^{(l)} R_{N-1}^{(l)} \ldots R_{2}^{(l)} R_{1}^{(l)} \quad \text { with } \quad l=2,3,4 S, 4 A
$$

The $R_{k}^{(2)}$ matrices all describe a $2 \mathrm{D}$ rotation of angle $\pi J \varepsilon$. Therefore:

$$
R^{(2)}=\left[\begin{array}{cc}
\cos (\pi J \tau) & -\sin (\pi J \tau) \\
\sin (\pi J \tau) & \cos (\pi J \tau)
\end{array}\right]
$$

As a consequence of the structure of the $R_{k}^{(4)}$ matrices, it is useful to notice that a product of such matrices, such as $R^{(4)}$, satisfies the

$$
R_{11}^{(4)}=R_{22}^{(4)} \quad \text { and } \quad R_{12}^{(4)}=-R_{21}^{(4)}
$$

matrix element equalities. Moreover,

$$
R_{12}^{(3)}=-R_{21}^{(3)}
$$

because the shape of the pulse envelope is symmetrical.

The two state evolutions of interest between $t_{2}$ and $t_{3}$ are the ones that transform -1 quantum states into +1 quantum states and vice versa. The corresponding state bases $\mathcal{B}^{(-)}$and $\mathcal{B}^{(+)}$can each be split into two subbases, one for the symmetrical and the other one for the antisymmetrical base element combinations:

$$
\begin{aligned}
& \mathcal{B}^{(-S)}=\left\{I_{-}+S_{-}, 2 I_{-} S_{z}+2 I_{z} S_{-}\right\} \\
& \mathcal{B}^{(-A)}=\left\{I_{-}-S_{-}, 2 I_{-} S_{z}-2 I_{z} S_{-}\right\} \\
& \mathcal{B}^{(+S)}=\left\{I_{+}+S_{+}, 2 I_{+} S_{z}+2 I_{z} S_{+}\right\} \\
& \mathcal{B}^{(+A)}=\left\{I_{+}-S_{+}, 2 I_{+} S_{z}-2 I_{z} S_{+}\right\}
\end{aligned}
$$

so that

$$
\mathcal{B}^{(-)}=\mathcal{B}^{(-S)} \cup \mathcal{B}^{(-A)} \quad \text { and } \quad \mathcal{B}^{(+)}=\mathcal{B}^{(+S)} \cup \mathcal{B}^{(+A)}
$$

Any linear combination of elements in $\mathcal{B}^{(-S)}$ is also a combination of elements in $\mathcal{B}^{(3)} \cup \mathcal{B}^{(4 S)}$. It is transformed by $\hat{\mathcal{H}}^{r}$ into another combination of elements in $\mathcal{B}^{(3)} \cup \mathcal{B}^{(4 S)}$. The projection of this state in the $\mathcal{B}^{(+)}$-generated subspace, as achieved by the gradient pulse at time $t_{3}$, is a combination of elements from $\mathcal{B}^{(+S)}$. A similar deduction indicates that a spin state from the $\mathcal{B}^{(-A)}$-generated subspace is transformed into a linear combination of elements in $\mathcal{B}^{(+A)}$. This means that the 
matrix of $\hat{\mathcal{H}}^{r}$ and of the gradient pulse pair has a block-diagonal structure, when written from base $\mathcal{B}^{(-)}$to base $\mathcal{B}^{(+)}$.

$$
M^{(+-)}=\left[\begin{array}{c|c}
M^{(S)} & 0 \\
\hline 0 & M^{(A)}
\end{array}\right]
$$

The elements in $M^{(S)}$ and $M^{(A)}$ are easily deduced from those in $R^{(2)}, R^{(3)}$, and $R^{(4)}$ :

$$
\begin{aligned}
& 2 M^{(S)}=\left[\begin{array}{cc}
R_{11}^{(3)}-R_{11}^{(4)} & i\left(R_{21}^{(3)}+R_{21}^{(4)}\right) \\
-i\left(R_{21}^{(3)}+R_{21}^{(4)}\right) & R_{11}^{(4)}-R_{22}^{(3)}
\end{array}\right] \\
& 2 M^{(A)}=\left[\begin{array}{cc}
R_{11}^{(2)}-R_{11}^{(4)} & i\left(R_{21}^{(2)}+R_{21}^{(4)}\right) \\
-i\left(R_{21}^{(2)}+R_{21}^{(4)}\right) & R_{11}^{(4)}-R_{11}^{(2)}
\end{array}\right]
\end{aligned}
$$

using properties 7, 8 and 9 . The matrix $M^{(-+)}$of the same transformation, but expressed from base $\mathcal{B}^{(+)}$to base $\mathcal{B}^{(-)}$is the complex conjugate of $M^{(-+)}$:

$$
M^{(-+)}=\left(M^{(+-)}\right)^{*}
$$

To fully describe what happens between times $t_{2}$ and $t_{3}$ to transverse magnetization it is also necessary to write the transformation matrices of

$$
\sigma \longrightarrow U(0) \sigma U^{-1}(0) \quad \text { and } \quad \sigma \longrightarrow U^{-1}(\tau) \sigma U(\tau)
$$

in the $\mathcal{B}^{(-)}$and $\mathcal{B}^{(+)}$bases (see Eq. 1). Indeed, these transformations are the same because

$$
U(0)=e^{-i \omega_{0}\left(I_{z}-S_{z}\right) \frac{\tau}{2}}=U^{-1}(\tau)
$$

The superoperator $\hat{U}$ that is defined by

$$
\hat{U}(\sigma)=U(0) \sigma U^{-1}(0)
$$

acts independently in bases $\mathcal{B}^{(-)}$and $\mathcal{B}^{(+)}$. More precisely, it acts independently in the $\mathcal{B}^{(-0)}, \mathcal{B}^{(-z)}, \mathcal{B}^{(+0)}$ and $\mathcal{B}^{(+z)}$ bases that are defined by:

$$
\begin{aligned}
& \mathcal{B}^{(-0)}=\left\{I_{-}+S_{-}, I_{-}-S_{-}\right\} \\
& \mathcal{B}^{(-z)}=\left\{2 I_{-} S_{z}+2 I_{z} S_{-}, 2 I_{-} S_{z}-2 I_{z} S_{-}\right\} \\
& \mathcal{B}^{(+0)}=\left\{I_{+}+S_{+}, I_{+}-S_{+}\right\} \\
& \mathcal{B}^{(+z)}=\left\{2 I_{+} S_{z}+2 I_{z} S_{+}, 2 I_{+} S_{z}-2 I_{z} S_{+}\right\}
\end{aligned}
$$

so that

$$
\mathcal{B}^{(-)}=\mathcal{B}^{(-0)} \cup \mathcal{B}^{(-z)} \quad \text { and } \quad \mathcal{B}^{(+)}=\mathcal{B}^{(+0)} \cup \mathcal{B}^{(+z)}
$$

In both $\mathcal{B}^{(-0)}$ and $\mathcal{B}^{(-z)}$ bases, the matrix of $\hat{U}$ is $S\left(\alpha_{0} / 2\right)$ in which the angle $\alpha_{0}$ 
and the matrix function $S$ are defined by:

$$
\begin{aligned}
\alpha_{0} & =\omega_{0} \tau \\
S(\varphi) & =\left[\begin{array}{cc}
\cos \varphi & i \sin \varphi \\
i \sin \varphi & \cos \varphi
\end{array}\right]
\end{aligned}
$$

Therefore, the matrix of $\hat{U}$ in $\mathcal{B}^{(-)}$is

$$
U^{(--)}=S\left(\alpha_{0} / 2\right) \otimes I_{2}
$$

in which $I_{2}$ is the $2 \times 2$ identity matrix. In a similar way, the matrix of $\hat{U}$ in $\mathcal{B}^{(+)}$ is

$$
U^{(++)}=S\left(-\alpha_{0} / 2\right) \otimes I_{2}=\left(U^{(--)}\right)^{*}
$$

With these results, the matrix of the spin state transformation between times $t_{2}$ and $t_{3}, R^{(+-)}$, can be expressed from base $\mathcal{B}^{(-)}$to base $\mathcal{B}^{(+)}$by

$$
R^{(+-)}=U^{(++)} M^{(+-)} U^{(--)}=\left(S\left(-\alpha_{0} / 2\right) \otimes I_{2}\right) M^{(+-)}\left(S\left(\alpha_{0} / 2\right) \otimes I_{2}\right)
$$

This expression is simplified by the indroduction of the $M^{(0)}$ and $E$ matrices:

$$
M^{(S)}=M^{(0)}+E \quad M^{(A)}=M^{(0)}-E
$$

It must be noticed that, in general, $M^{(S)} \neq M^{(A)}$. This means that $E \neq 0$ and therefore that $I_{-}+S_{-}$in not transformed between times $t_{2}$ and $t_{3}$ in the same way as $I_{-}-S_{-}$. The matrix $E$ characterizes a sort of ellipticity in the behavior of the soft pulse, hence its name. Using the matrix function $A$ :

$$
A(\varphi)=\left[\begin{array}{cc}
\cos \varphi & i \sin \varphi \\
-i \sin \varphi & -\cos \varphi
\end{array}\right]
$$

$M^{(+-)}$is rewritten as

$$
M^{(+-)}=I_{2} \otimes M^{(0)}+A(0) \otimes E
$$

Then

$$
\begin{aligned}
R^{(+-)} & =I_{2} \otimes M^{(0)}+\left(S\left(-\alpha_{0} / 2\right) \otimes I_{2}\right)(A(0) \otimes E)\left(S\left(\alpha_{0} / 2\right) \otimes I_{2}\right) \\
& =I_{2} \otimes M^{(0)}+A\left(\alpha_{0}\right) \otimes E
\end{aligned}
$$

because

$$
S(-\psi) S(\psi)=I_{2} \quad \text { and } \quad S(-\psi) A(\varphi) S(\psi)=A(\varphi+2 \psi)
$$

As usual, the transformation matrix of +1 quantum states to -1 quantum state, is the complex conjugate of $R^{(+-)}$:

$$
R^{(-+)}=U^{(--)} M^{(-+)} U^{(++)}=\left(R^{(+-)}\right)^{*}
$$




\subsection{Between $t_{1}$ and $t_{4}$}

The action of $H_{0}$ during the $\delta$ delays can be divided into two commutating parts, one related to nuclei offsets and the other to their mutual coupling. The former achieves the transformation

$$
\sigma \longrightarrow e^{-i \omega_{0}\left(I_{z}-S_{z}\right) \delta} \sigma e^{i \omega_{0}\left(I_{z}-S_{z}\right) \delta}
$$

that can be merged with the similarity transformation by replacing $\alpha_{0}$ by $\alpha$ :

$$
\alpha=\alpha_{0}+2 \omega_{0} \delta=\omega_{0}(\tau+2 \delta)=\omega_{0} \Delta / 2
$$

The coupling operator acts identically in $\mathcal{B}^{(-S)}$ and $\mathcal{B}^{(-A)}$, as well as in $\mathcal{B}^{(+S)}$ and $\mathcal{B}^{(+A)}$. The corresponding superoperator is respectively expressed in $\mathcal{B}^{(-)}$and $\mathcal{B}^{(+)}$ by the matrices

$$
D^{(--)}=I_{2} \otimes S(\beta / 2) \quad \text { and } \quad D^{(++)}=I_{2} \otimes S(-\beta / 2)=\left(D^{(--)}\right)^{*}
$$

in which the $\beta$ angle is defined by

$$
\beta=2 \pi J \tau
$$

Therefore, the matrix $R^{(+-)}(\delta)$ of the superoperator that acts between times $t_{1}$ and $t_{4}$ from $\mathcal{B}^{(-)}$to $\mathcal{B}^{(+)}$is:

$$
\left.\left.R^{(+-)}(\delta)=\left(S(-\alpha / 2) \otimes I_{2}\right)\right)\left(D^{(++)} M^{(+-)} D^{(--)}\right)\left(S(\alpha / 2) \otimes I_{2}\right)\right)
$$

Considering that both the $M^{(0)}$ and $E$ matrices look like

$$
M=\left[\begin{array}{cc}
u & i v \\
-i v & w
\end{array}\right]
$$

$u, v$ and $w$ being three real numbers and that

$$
\begin{aligned}
M & =C I_{2}+F A(\theta) \\
\text { with } \quad C & =\frac{1}{2} \operatorname{tr}(M) \\
z & =\frac{u-w}{2}+i v \\
F & =|z| \\
\text { and } \quad \theta & =\arg (z)
\end{aligned}
$$

the matrices $M^{(0)}$ and $E$ can be rewritten as

$$
M^{(0)}=C_{0} I_{2}+F_{0} A\left(\theta_{0}\right) \text { and } E=C_{e} I_{2}+F_{e} A\left(\theta_{e}\right) .
$$

Indeed, $\operatorname{tr}\left(M^{(0)}\right)=\operatorname{tr}(E)$ because the $M^{(A)}$ matrix is traceless, according to Eq. 11. The $C_{0}$ and $C_{e}$ coefficients are therefore equal and simply noted $C$. Consequently,

$$
\begin{aligned}
S(-\beta / 2) M^{(0)} S(\beta / 2) & =C I_{2}+F_{0} A\left(\beta_{0}\right) \\
S(-\beta / 2) E S(\beta / 2) & =C I_{2}+F_{e} A\left(\beta_{e}\right)
\end{aligned}
$$


with

$$
\beta_{0}=\beta+\theta_{0} \quad \text { and } \quad \beta_{e}=\beta+\theta_{e}
$$

The insertion of Eqs. 15 and 16 in Eq. 13 gives

$$
R^{(+-)}(\delta)=I_{2} \otimes\left(C I_{2}+F_{0} A\left(\beta_{0}\right)\right)+A(\alpha) \otimes\left(C I_{2}+F_{e} A\left(\beta_{e}\right)\right)
$$

This equation is the central and most important result of this article. It shows that the effect of the single echo sequence between times $t_{1}$ and $t_{4}$ depends on the difference of the resonance frequencies of nuclei I and S, something that is unexpected from a spin echo sequence. The dependence is introduced through the transverse magnetization precession angle $\alpha$, whose value is $\omega_{0} \Delta / 2$, and only in relation to the soft pulse ellipticity parameters $F_{e}$ and $\theta_{e}$.

As usual,

$$
R^{(-+)}(\delta)=\left(R^{(+-)}(\delta)\right)^{*}
$$

It should also be noted that $R^{(+-)}(\delta)$ and $R^{(-+)}(\delta)$ are Hermitian matrices.

\subsection{Between $t_{1}$ and $t_{5}$}

The -1 and +1 quantum states evolve between $t_{1}$ and $t_{5}$ according to the double spin echo transmission matrices $T^{(--)}$and $T^{(++)}$:

$$
T^{(--)}=R^{(-+)}(\delta) R^{(+-)}(\delta) \quad T^{(++)}=R^{(+-)}(\delta) R^{(-+)}(\delta) .
$$

The only base elements in $\mathcal{B}^{(-)}$that are able to produce double quantum states at $t_{6}$ are the coupled states whose indexes in $\mathcal{B}^{(-)}$are 2 and 4 . Thus, the terms of interest in $T^{(--)}$are the matrix elements $T_{21}^{(--)}$and $T_{41}^{(--)}$, index 1 being that of the $I_{-}+S_{-}$state. According to Eq. 18 and to the Hermiticity of $R^{(-+)}(\delta)$ :

$$
\begin{aligned}
T_{21}^{(--)} & =\sum_{k=1}^{4} R_{2 k}^{(-+)}(\delta) R_{k 1}^{(+-)}(\delta) \\
& =\sum_{k=1}^{4}\left(R_{k 2}^{(-+)}(\delta)\right)^{*} R_{k 1}^{(+-)}(\delta) \\
& =\sum_{k=1}^{4} R_{k 2}^{(+-)}(\delta) R_{k 1}^{(+-)}(\delta)
\end{aligned}
$$

This shows that $T_{21}^{(--)}$is the scalar product of $R_{\bullet 1}^{(+-)}(\delta)$ and $R_{\bullet 2}^{(+-)}(\delta)$, the first and second column in $R^{(+-)}(\delta)$ :

$$
R_{\bullet 1}^{(+-)}(\delta)=\mid \begin{aligned}
& \left(C+F_{0} \cos \beta_{0}\right)+\cos \alpha\left(C+F_{e} \cos \beta_{e}\right) \\
& -i\left(F_{0} \sin \beta_{0}+\cos \alpha F_{e} \sin \beta_{e}\right) \\
& -i \sin \alpha\left(C+F_{e} \cos \beta_{e}\right) \\
& -\sin \alpha F_{e} \sin \beta_{e}
\end{aligned}
$$


and

$$
R_{.2}^{(+-)}(\delta)=\mid \begin{aligned}
& i\left(F_{0} \sin \beta_{0}+\cos \alpha F_{e} \sin \beta_{e}\right) \\
& \left(C-F_{0} \cos \beta_{0}\right)+\cos \alpha\left(C-F_{e} \cos \beta_{e}\right) \\
& \sin \alpha F_{e} \sin \beta_{e} \\
& -i \sin \alpha\left(C-F_{e} \cos \beta_{e}\right)
\end{aligned}
$$

from which

$$
\begin{aligned}
T_{21}^{(--)} / i= & F_{0}^{2} \sin \left(2 \beta_{0}\right) \\
& +2 F_{0} F_{e} \cos \alpha \sin \left(\beta_{0}+\beta_{e}\right) \\
& +F_{e}^{2} \cos (2 \alpha) \sin \left(2 \beta_{e}\right) \\
T_{21}^{(++)}= & -T_{21}^{(--)}
\end{aligned}
$$

A similar calculation shows that $T_{41}^{(--)}=T_{41}^{(++)}$is a real number whose value is not relevant, as shown hereafter. When taking into account only the antiphase magnetization that is produced between times $t_{1}$ and $t_{5}$, the preceding results are summarized by:

$$
\begin{aligned}
& I_{-}+S_{-} \longrightarrow T_{21}^{(--)}\left(2 I_{-} S_{z}+2 I_{z} S_{-}\right)+T_{41}^{(--)}\left(2 I_{-} S_{z}-2 I_{z} S_{-}\right) \\
& I_{+}+S_{+} \longrightarrow-T_{21}^{(--)}\left(2 I_{-} S_{z}+2 I_{z} S_{-}\right)+T_{41}^{(--)}\left(2 I_{-} S_{z}-2 I_{z} S_{-}\right)
\end{aligned}
$$

from which the evolution of the initial transverse magnetization

$$
\sigma_{1}=I_{x}+S_{x} \longrightarrow \sigma_{5}=T_{21}^{(--)} / i\left(2 I_{y} S_{z}+2 I_{z} S_{y}\right)+T_{41}^{(--)}\left(2 I_{x} S_{z}+2 I_{z} S_{x}\right)
$$

is deduced.

\subsection{Between $t_{1}$ and $t_{6}$}

The second hard pulse of the sequence is only able to convert the first term of $\sigma_{5}$ (Eq. 19) into the $\sigma_{6}$ double quantum state:

$$
\sigma_{6}=\left(2 I_{y} S_{x}+2 I_{x} S_{y}\right)
$$

This state is the only one to be converted back to antiphase magnetization by the last hard pulse of the sequence. Subsequent evolution during the detection period yields a signal whose Fourier transform is an antiphase doublet whose intensity is proportional to

$$
f=T_{21}^{(--)} / i
$$

where the "transmission factor" $f$ is the value to be optimized, as a function of the $\delta$ delay .

The analytical expression of $f$ (Eqs. 19 and 21) can be expanded to explicitly show how it depends on the $4 \delta$ and $2 \tau$ pulse sequence parameters, on the $F_{0}, \theta_{0}$, 
$F_{e}, \theta_{e}$ soft pulse parameters, and on the $J$ and $\Delta \nu$ spin system parameters.

$$
\begin{aligned}
f(\delta)= & F_{0}^{2} \sin \left(\pi J(4 \delta)+2 \theta_{0}\right) \\
& +2 F_{0} F_{e} \cos (\pi \Delta \nu(4 \delta+2 \tau) / 2) \sin \left(\pi J(4 \delta)+\theta_{0}+\theta_{e}\right) \\
& +F_{e}{ }^{2} \cos (\pi \Delta \nu(4 \delta+2 \tau)) \sin \left(\pi J(4 \delta)+2 \theta_{e}\right) .
\end{aligned}
$$

The $\delta$ value that maximises $f(\delta)$ is certainly best obtained by plotting $f$ as a function of $\delta$. For this purpose a computer programme, named Bisel, is available at http://www.univ-reims.fr/LSD/JmnSoft/Bisel, even though calculations could, in principle, be achieved by any spin dynamics simulator that handles static field gradient pulses.

\section{Experimental}

Spectra were recorded on a Bruker DRX spectrometer operating at $500.13 \mathrm{MHz}$ for the ${ }^{1} \mathrm{H}$ nucleus. The pulse sequence in Fig. 1 was applied to a sample made of $10 \mathrm{mg}$ of cinnamic acid dissolved in $0.7 \mathrm{~mL}$ of deuterated chloroform. The two ethylenic protons at 7.86 and 6.53 share a single scalar coupling of intensity $J=15.96 \mathrm{~Hz}$. Their resonance frequency difference $\Delta \nu=669.2 \mathrm{~Hz}$ was high enough relatively to $J$ to consider that theses nuclei form an $A X$ spin system. For each pulse sequence a set of 512 FIDs was recorded, in which $4 \delta$ was regularly incremented from 0 to $1 / J$. The duration of the hard $90^{\circ}$ and soft $180^{\circ}$ pulses were $10.1 \mu$ s and 50 ms, respectively. Each FID was recorded by co-adding 8 transients separated by 5 seconds relaxation delays. The soft pulse envelope was a $1 \%$ truncated Gaussian that was amplitude modulated to achieve the desired double refocusing profile. Gradient pulse strenghs $G_{1}$ and $G_{2}$ were 38 and $10 \mathrm{G} . \mathrm{cm}^{-1}$. Each gradient pulse lasted $1 \mathrm{~ms}$ and was followed by a $100 \mu$ s recovery delay.

Raw data Fourier transformation was achieved without any apodization. All spectra were identically phase corrected to present pairs of pure absorption antiphase doublets. Experimental transmission factors were extracted by integration of the highest frequency peak of the spectra.

The simulation of the sequence in Fig. 1 by the Bisel computer programme used Eqs. $2-6,10,11,12,14$ and 22.

\section{Results and Discussion}

The experimental result in Fig. 2a is in very good agreement with the theoretical one (from Eq. 22), as visible in the graphical representation of the $f$ function in Fig. $2 \mathrm{~b}$. The antiphase magnetization production factor $f$ is the sum of three slow oscillating terms, all of frequency $J / 2$ if the length of the total additional delay $4 \delta$ is taken as the time variable of interest. The second and third terms in the expression of $f$ are modulated by high frequency oscillations. The modulation frequency of the second term is $\Delta \nu / 4$, while the one of the third term is $\Delta \nu / 2$. The amplitude of the rapid oscillations increases with the ellipticity parameter $F_{e}$.

The behaviour change of such a spin echo sequence according to the difference of nucleus resonance frequency $\Delta \nu$ was unexpected at the time this work was undertaken. This phenomenon was not previously reported. It is clearly related to the existence of non-identical transformation rules for $I_{-}+S_{-}$and for $I_{-}-S_{-}$states by action of $\hat{\mathcal{H}}^{r}$ between $t_{2}$ and $t_{3}$ (see Eq. 12). This means that a $I_{-}$state alone evolves to produce a $S_{-}$state, or, in other words, that the biselective refocusing 


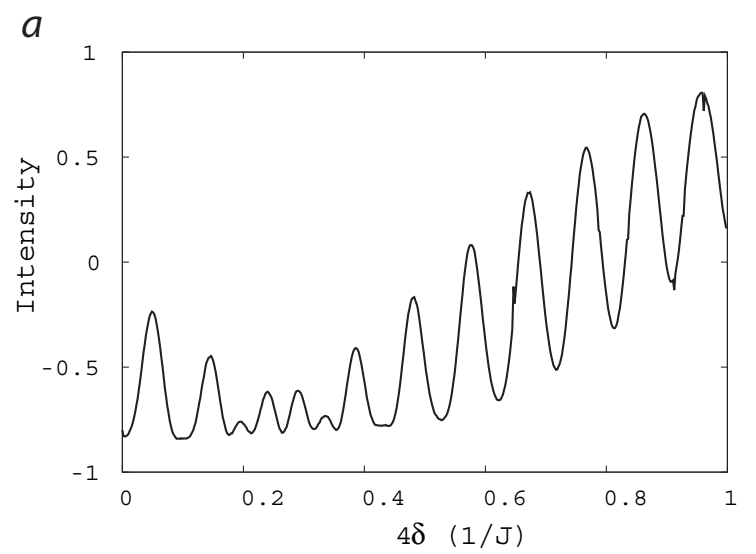

$b$

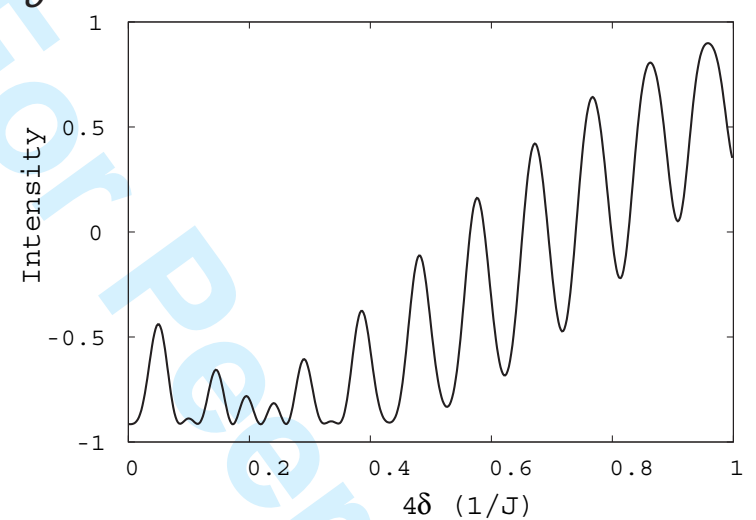

Figure 2. Plots of antiphase magnetization intensity as a function of $4 \delta$, expressed in $1 / J$ units. (a) Experimental values. The vertical scale is in arbitrary units but was chosen to roughly match the vertical scale in Fig. 2b. (b) Theoretical prediction, according to Eq. 22 and the Bisel computer programme.

pulses are able to induce a magnetization transfer between coupled nuclei. Such a transfer is conceptually not far from a homonuclear Hartmann-Hahn transfert [8]. The latter is caused by a radiofrequency field that is simultaneously applied to the transverse magnetization of a set of coupled spins, a situation that is similar to the present one.

The cinnamic acid example that is described here leads to

$$
\begin{array}{ll}
F_{0}=0.916 & \theta_{0}=110^{\circ} \\
F_{e}=0.275 & \theta_{e}=20^{\circ}
\end{array}
$$

parameter values, as calculated by the Bisel computer programme. In this context, $\delta=0$ ensures a theoretical signal "yield" of $91 \%$ with minimal signal loss through transverse relaxation.

Obviously, the pulse sequence in Fig. 1 was not designed to be applied solely to an isolated AX spin system. Numerical simulations show that passive couplings of I or $\mathrm{S}$ do not dramatically change the appearance of the graph of the $f(\delta)$ function. Multiplet widening causes signal loss but the slow and rapid oscillations occur at the same frequencies. Therefore, simulation of the pulse sequence according to the two-spin model offers a good starting point for more complex experiments.

\section{Conclusion}

The spin dynamics behind biselective gradient enhanced double spin echo NMR experiments is not as simple as it appears. The creation of antiphase magnetisation 
does not follow a simple law like $\sin (4 \pi J \delta+\phi)$ - the first term in Eq. 22 - but depends on the difference of nucleus resonance frequencies, in a way that can be analytically described. New applications of biselective pulses will be reported in the near future and their optimization will certainly benefit from this theoretical study.

\section{Acknowledgment}

I thank Dr. K. Plé for linguistic advice.

\section{References}

[1] L. Emsley, I. Burghardt, G. Bodenhausen. Double selective inversion in NMR and multiple quantum effects in coupled spin systems, J. Magn. Reson., 90, 214 (1990).

[2] J.-M. Nuzillard, R. Freeman. Correlation spectroscopy with two simultaneous soft pulses (TSETSE). J. Magn. Reson. A, 112, 72 (1995).

[3] V. Blechta, J. Schraml. Selective on-resonance double pulses applied to an IS spin system. J. Magn. Reson. A, 112, 30 (1995).

[4] V. Zhou, C. Ye, B.C. Sanctuary. Response of a coupled two-spin system to on-resonance amplitude modulated RF pulses. Mol. Phys., 87, 679 (1996).

[5] T.-L. Hwang, A.J. Shaka. Water suppression that works. Excitation sculpting using arbitrary waveforms and pulsed field gradients. J. Magn. Reson. A, 112, 275 (1995).

[6] S. Bourg, J.-M. Nuzillard. In phase double selective exitation of coupled spin systems using excitation sculpting. J. Magn. Reson., 133, 173 (1998).

[7] J. Cavanagh, W.J. Fairbrother, A.G. Palmer III, N.J. Skelton. itshape Protein NMR Spectroscopy: Principles and Practice, Academic Press, San Diego (1996).

[8] L. Braunschweiler, R. R. Ernst. Coherence transfer by isotropic mixing: application to proton correlation spectroscopy. J. Magn. Reson., 53, 521 (1983). 
$a$

\section{Page 15 of Mbolecular Physics}

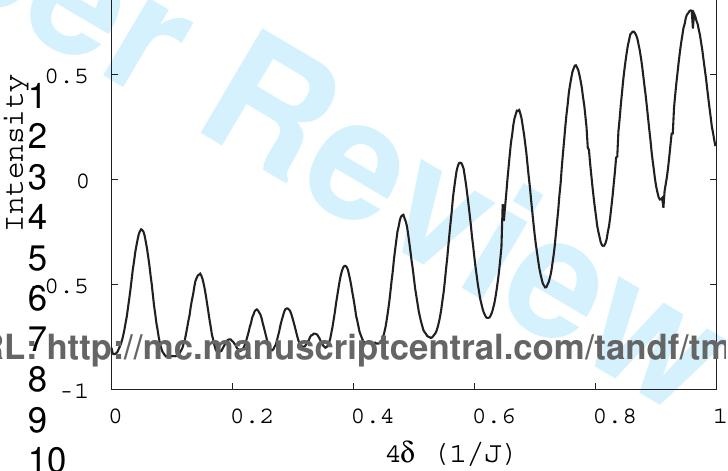




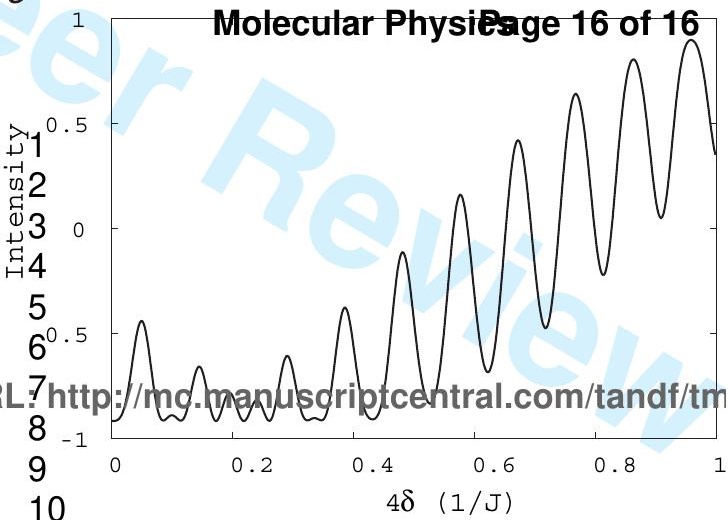

